\title{
Hybrid Precoding Design for SWIPT Joint Multicast-Unicast mmWave System with Subarray Structure
}

\author{
Wanming $\mathrm{Hao}^{1,2}$, Zheng Chu ${ }^{1}$, Fuhui Zhou ${ }^{3}$, Pei Xiao ${ }^{1}$, Victor C. M. Leung ${ }^{4}$, Rahim Tafazolli ${ }^{1}$ \\ ${ }^{1} 5 \mathrm{G}$ Innovation Centre, Institute of Communication Systems, University of Surrey, Guildford GU2 7XH, U.K. \\ ${ }^{2}$ School of Information Engineering, Zhengzhou University, Zhengzhou, China. \\ ${ }^{3}$ Department of Electrical and Computer Engineering, Utah State University, Utah, USA. \\ ${ }^{4}$ University of British Columbia, Vancouver, BCV6T 1Z4, Canada. \\ E-mail:\{w.hao, zheng.chu, p.xiao, r.tafazolli\}@surrey.ac.uk, zhoufuhui@ieee.org, vleung@ece.ubc.ca
}

\begin{abstract}
In this paper, we investigate the hybrid precoding design for joint multicast-unicast millimeter wave (mmWave) system, where the simultaneous wireless information and power transform is considered at receivers. The subarray-based sparse radio frequency chain structure is considered at base station (BS). Then, we formulate a joint hybrid analog/digital precoding and power splitting ratio optimization problem to maximize the energy efficiency of the system, while the maximum transmit power at BS and minimum harvested energy at receivers are considered. Due to the difficulty in solving the formulated problem, we first design the codebook-based analog precoding approach and then, we only need to jointly optimize the digital precoding and power splitting ratio. Next, we equivalently transform the fractional objective function of the optimization problem into a subtractive form one and propose a two-loop iterative algorithm to solve it. For the outer loop, the classic Bi-section iterative algorithm is applied. For the inner loop, we transform the formulated problem into a convex one by successive convex approximation techniques, which is solved by a proposed iterative algorithm. Finally, simulation results are provided to show the performance of the proposed algorithm.
\end{abstract}

Index Terms-Hybrid precoding, mmMave, Multicast, Unicast, Energy efficiency, SWIPT.

\section{INTRODUCTION}

Millimeter wave (mmWave) (30-300 GHz), owning the wider bandwidth, has been considered as a promising technique to meet the requirement with an exponential data traffic growth in future wireless communications [1]. Furthermore, due to the short wavelengths in mmWave bands, more antennas can be packed with a small physical size [2]. However, the use of a large number of antennas will cause a huge energy consumption and hardware cost when fully digital signal processing is applied, in the sense that each antenna needs a dedicate radio frequency (RF) chain, and the power consumption of the RF chain is as high as $250 \mathrm{~mW}$ at mmWave frequencies [3]. To reduce the energy consumption, a hybrid analog/digital precoding scheme can be employed, where the required number of RF chains will be much less than that of antennas [4].

On the other hand, the simultaneous wireless information and power transfer (SWIPT) has also been identified a promising technique for future wireless communications [5]. With power splitting, the receivers split the received RF signals for information detection and energy harvesting at the same time [6]. In fact, SWIPT is a very effective solution for a multiuser system, where the interference power can be transformed into the energy at receivers. However, the interference channel also causes the difficulty for information decoding. Therefore, how to trade off the information and harvested energy is a challenge in the SWIPT system. In addition, there is an increasing demand for video transmissions and TV broadcasting over cellular networks, where multiple users intend to receive the same content [7]. Meanwhile, each user still wishes to receive the private message, for example, in the object oriented broadcasting (OBB) scenario. As a result, the joint multicast and unicast transmission can be an effective scheme to satisfy the users for simultaneously receiving the private and common messages, and in the meantime, performance enhancement can be achieved in comparison with the conventional frequency/time division multiplexing [8].

Currently, some works have been conducted to address the above problem. For the SWIPT system, [9] studies the joint optimal power allocation and power splitting ratio to maximize the minimum signal to interference plus noise ratio (SINR) of all users. The authors in [10] apply the SWIPT to a multicast system, where multiple users share the same message. The authors propose an efficient subcarrier allocation and power allocation scheme to maximize the minimum SINR at each subcarrier. Similarly, they later formulate a nonconvex optimization problem as maximizing the minimum SINR among users in [11], and two successive convex approximation (SCA)based iterative algorithms are proposed to solve the formulated problem. Meanwhile, they extend the system to the sparse RF chain structure at BS in [12]. For the above system, they develop an efficient antenna selection and hybrid beamforming design algorithm to minimize the transmit power. In addition, [13] investigates the spectrum and energy-efficient beamforming design problem in the mMIMO-NOMA mmWave with lens antenna array, and the $\mathrm{ZF}$ precoding scheme is used to reduce the inter-beam interference. Then, a dynamic power allocation algorithm is proposed to maximize the sum rate of the system. However, the existing works have not jointly investigated the multicast-unicast mmWave communication with SWIPT.

Different from the previous works, in this paper, we consider the joint multicast-unicast mmWave communication, where the SWIPT is applied at each receiver. To reduce the hardware cost and energy consumption, we consider the sparse RF chain structure at BS. Based on the above considered system, we formulate an energy efficiency (EE) maximization problem by jointly optimizing hybrid analog/digital precoding and power 


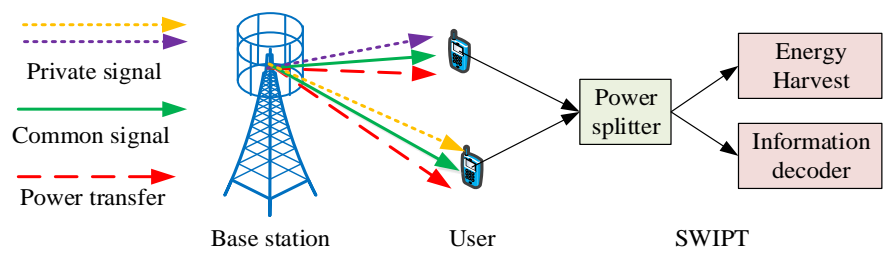

Fig. 1: Multicast-unicast mmWave communications with SWIPT.

splitting ratio. Meanwhile, we consider the maximum transmit power constraint for the BS and the minimum harvested energy requirement for each receiver. The formulated problem is a nonconvex optimization problem, which is intractable in its original form. Based on this, we first propose a codebook-based analog precoding. Next, we still need to jointly optimize the digital precoding and power splitting ratio, and we equivalently transform the fractional objective function of the optimization problem into a subtractive form one and a two-loop iterative algorithm is developed to solve it. Specifically, the Bi-section iterative algorithm is applied at outer loop. However, for inner loop, we need to solve a nonconvex optimization problem. To this end, by introducing new variables and employing the SCA technique, we transform the original nonconvex optimization problem into a convex one and propose an iterative algorithm to solve it.

Notations: We use the following notations throughout this paper: $(\cdot)^{*},(\cdot)^{T}$ and $(\cdot)^{H}$ denote the conjugate, transpose and Hermitian transpose, respectively, $\|\cdot\|$ is the Frobenius norm, $\mathbb{C}^{x \times y}$ means the space of $x \times y$ complex matrix, $\operatorname{Re}(\cdot)$ denotes real number operation.

\section{System Description And Problem Formulation}

We consider a downlink mmWave communication system as shown in Fig. 1, where the BS is equipped with $N_{\mathrm{TX}}$ antennas. To reduce the hardware cost and power consumption, we assume that the BS is equipped with $N_{\mathrm{RF}} \mathrm{RF}$ chains $\left(N_{\mathrm{RF}} \leq N_{\mathrm{TX}}\right)$. In addition, $K\left(K \leq N_{\mathrm{RF}}\right)$ single-antenna users are served simultaneously with multicast and unicast, and $\mathcal{K}=\{1, \ldots, K\}$ denotes the set of all users. In this paper, we focus on a scenario that all users receive a common information stream by broadcast, meanwhile each user obtains a private information stream by unicast. For the subarray structure at BS, each RF chain is connected to a disjoint subset of antennas with several phase shifters as shown in Fig. 2.

The received signal by the $k$ th user can be represented as

$$
y_{k}=\mathbf{h}_{k} \mathbf{F} \mathbf{v}_{k} x_{k}+\mathbf{h}_{k} \mathbf{F} \mathbf{v}_{0} x_{0}+\mathbf{h}_{k} \mathbf{F} \sum_{i \neq k}^{K} \mathbf{v}_{i} x_{i}+n_{k},
$$

where $\mathbf{h}_{k} \in \mathbb{C}^{1 \times N_{\mathrm{TX}}}, \mathbf{v}_{k} \in \mathbb{C}^{N_{\mathrm{RF}} \times 1}$ and $x_{k}$, respectively, denote the downlink channel, digital precoding and private signal for the $k$ th user. $\mathbf{v}_{0} \in \mathbb{C}^{N_{\mathrm{RF}} \times 1}$ and $x_{0}$ are the digital precoding and common signal for the $k$ th user, respectively. $n_{k}$ is an independent and identically distributed (i.i.d.) additive white Gaussian noise (AWGN) defined as $C \mathcal{N}\left(0, \delta_{0}^{2}\right)$. F $\in \mathbb{C}^{N_{\mathrm{TX}} \times N_{\mathrm{RF}}}$ means the analog

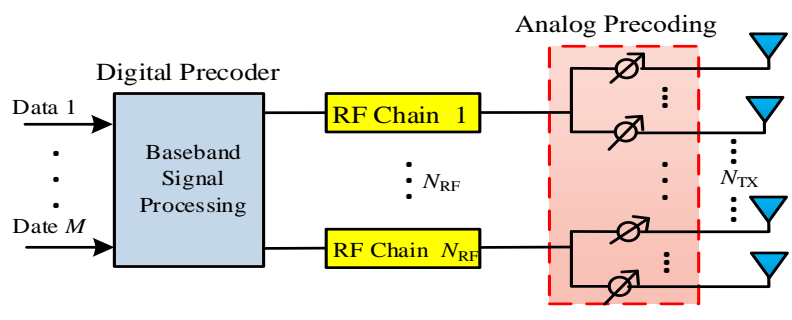

Fig. 2: Subarray structure antenna

precoding matrix implemented by the equal power splitter and phase shifters [14], which can be represented as

$$
\mathbf{F}=\left[\begin{array}{cccc}
\mathbf{f}_{1} & \mathbf{0} & \cdots & \mathbf{0} \\
\mathbf{0} & \mathbf{f}_{2} & \cdots & \mathbf{0} \\
\vdots & \vdots & \ddots & \vdots \\
\mathbf{0} & \mathbf{0} & \cdots & \mathbf{f}_{N_{\mathrm{RF}}}
\end{array}\right]
$$

where $\mathbf{f}_{k} \in \mathbb{C}^{N_{\mathrm{SUB}} \times 1}$ denotes the precoding vector associated with the $k$-th RF chain with $\left|\left(\mathbf{f}_{k}\right)_{i}\right|=1 / \sqrt{N_{\text {SUB }}}\left(i=1, \ldots, N_{\mathrm{SUB}}\right)$. Here, $N_{\text {SUB }}$ denotes the number of antennas connected to each $\mathrm{RF}$ chain, and we assume that $N_{\mathrm{SUB}}$ is the same for all RF chains with $N_{\mathrm{SUB}}=N_{\mathrm{TX}} / N_{\mathrm{RF}}{ }^{1}$.

In addition, each user splits the received signal into information decoder (ID) and energy harvester (EH). We assume that the $\beta_{k}$ portion of received signal power is divided into the ID, while the remaining $1-\beta_{k}$ portion of the received signal power is transformed into the EH. Accordingly, the received signal used for EH by the $k$ th user can be written as

$$
y_{k}^{\mathrm{EH}}=\sqrt{1-\beta_{k}} y_{k},
$$

and the harvested energy is

$$
E_{k}=\varepsilon\left(1-\beta_{k}\right)\left(\sum_{i=0}^{K}\left|\mathbf{h}_{k} \mathbf{F} \mathbf{v}_{i}\right|^{2}+\delta_{0}^{2}\right),
$$

where $\varepsilon \in(0,1]$ denotes the energy conversion efficiency. The received signal used for ID can be represented as

$$
y_{k}^{\mathrm{ID}}=\sqrt{\beta_{k}} y_{k}+n_{k}^{\prime},
$$

where $n_{k}^{\prime} \sim \mathcal{C N}\left(0, \delta_{1}^{2}\right)$ is the addition noise caused by the ID.

By using the layered division multiplexing, the common and private signal can be transmitted simultaneously [15]. At receiver, the user can decode the signals with successive interference cancellation. Based on this, each user first decodes the common signal by treating all private signals as noise ${ }^{2}$, and then the private signal is decoded by subtracting the common signal from the received signal. To this end, the achievable SINR of the common signal at the $k$ th user can be expressed as

$$
\gamma_{k}^{0}=\frac{\beta_{k}\left|\mathbf{h}_{k} \mathbf{F} \mathbf{v}_{0}\right|^{2}}{\beta_{k}\left(\sum_{i=1}^{K}\left|\mathbf{h}_{k} \mathbf{F} \mathbf{v}_{i}\right|^{2}+\delta_{0}^{2}\right)+\delta_{1}^{2}},
$$

and the achievable SINR of the private signal at the $k$ th user

\footnotetext{
${ }^{1}$ Here, $N_{\text {SUB }}$ should be an integer. In fact, when $N_{\text {SUB }}$ is not an integer, i.e. the number of antennas in different subarrays may be different, it is also suitable in our scheme.

${ }^{2}$ In general, the multicast message have a higher priority because it is sent to multiple users [15]. Therefore, we assume that the common signal is first decoded.
} 
can be represented as

$$
\gamma_{k}=\frac{\beta_{k}\left|\mathbf{h}_{k} \mathbf{F} \mathbf{v}_{k}\right|^{2}}{\beta_{k}\left(\sum_{i \neq k}^{K}\left|\mathbf{h}_{k} \mathbf{F} \mathbf{v}_{i}\right|^{2}+\delta_{0}^{2}\right)+\delta_{1}^{2}} .
$$

For mmWave channel, we adopt a widely used geometric channel mode as follows [16],

$$
\mathbf{h}_{k}=\sqrt{\frac{N_{\mathrm{TX}}}{\rho_{k}}} \sum_{l=1}^{L} \alpha_{k}^{l} \mathbf{a}^{H}\left(\theta_{k}^{l}\right),
$$

where $\rho_{k}$ denotes the average path-loss from the BS to the $k$ th user, and $\alpha_{k}$ represents the complex gain of the $l$ th path. $\mathbf{a}\left(\theta_{k}^{l}\right)$ is the antenna array response vector at user $k$. When the uniform linear array is used, $\mathbf{a}\left(\theta_{k}^{l}\right)$ can be expressed as

$$
\mathbf{a}\left(\theta_{k}^{l}\right)=\frac{1}{\sqrt{N_{\mathrm{TX}}}}\left[1, e^{j(2 \pi / \lambda) d \sin \left(\theta_{k}^{l}\right)}, \ldots, e^{j\left(N_{\mathrm{TX}}-1\right)(2 \pi / \lambda) d \sin \left(\theta_{k}^{l}\right)}\right]^{T},
$$

where $\theta_{k}^{l} \in[0,2 \pi]$ is the azimuth angles of departure of the BS at the $l$ th path, $\lambda$ is the wavelength, and $d$ is the distance between two adjacent antenna array elements.

In general, the power consumption includes two parts, namely transmit power and circuit power consumption. The circuit power consumption mainly includes baseband signal processing, RF chains and phase shifters [2]. The circuit power consumptions can be written as

$$
P_{\mathrm{C}}=P_{\mathrm{BB}}+N_{\mathrm{RF}} P_{\mathrm{RF}}+N_{\mathrm{TX}} P_{\mathrm{PS}},
$$

where $P_{\mathrm{BB}}, P_{\mathrm{RF}}$, and $P_{\mathrm{PS}}$, respectively, denote the power consumption of the baseband, the RF chain and the phase shifter. Finally, we give the total power consumption as follows

$$
P_{\text {total }}=\sum_{k=0}^{K} \xi\left\|\mathbf{F} \mathbf{v}_{k}\right\|^{2}+P_{\mathrm{C}} .
$$

where $\xi \geq 1$ is the inefficiency of the power amplifier.

Next, we define the EE of the system as [17]

$$
\eta_{\text {EE }}=\frac{\min _{\forall k}\left\{\log _{2}\left(1+\gamma_{k}^{0}\right)\right\}+\sum_{k=1}^{K} \log _{2}\left(1+\gamma_{k}\right)}{P_{\text {total }}} .
$$

In this paper, we aim to maximize the EE of the system by jointly optimizing the power splitting ratio $\beta_{k}(k \in \mathcal{K})$, analog precoding $\mathbf{F}$ and digital precoding $\mathbf{v}_{k}(k \in\{0, \mathcal{K}\})$, which is written as

$$
\begin{array}{ll} 
& \max _{\left\{\left\{\beta_{k}\right\}, \mathbf{F},\left\{\mathbf{v}_{k}\right\}\right\}} \quad \eta_{\mathrm{EE}} \\
\text { s.t. } & E_{k} \geq E_{k}^{\min }, k \in \mathcal{K}, \\
& \sum_{k=0}^{K}\left\|\mathbf{F} \mathbf{v}_{k}\right\|^{2} \leq P_{\max },
\end{array}
$$

where (13b) denotes the minimum requirement of the harvested energy for each user, and (13c) defines the maximum transmit power constraint for the BS.

\section{Proposed Hybrid Precoding Algorithm}

\section{A. Analog Precoding Design}

For the sparse RF chain structure, the design of the analog precoding depends on the phase shifters. Since only quantized angles are available for the phase shifters, the selection of the precoding angles should depend on the finite-size codebook, and we assume that the codebook have the same form as the array steering vectors in (9). Based on this, we can obtain the analog precoding by searching the codebook. We define the

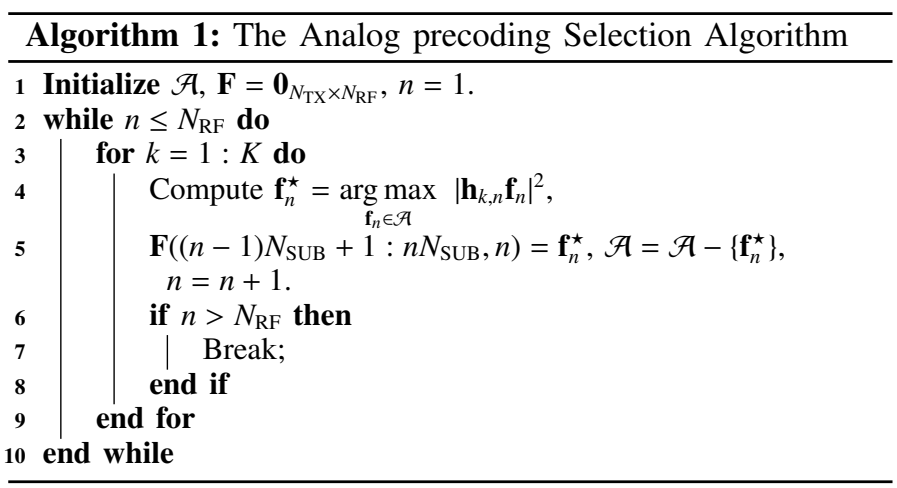

codebook as $\mathcal{A}=\left\{\mathbf{a}\left(\theta_{k}^{l}\right), \forall k, l\right\}$. For the subarray structure, all RF chains are connected to the disjoint subset of antennas, and each of them has one beamforming direction. To this end, we divide the channel $\mathbf{h}_{k}$ as $\mathbf{h}_{k}=\left[\mathbf{h}_{k, 1}, \mathbf{h}_{k, 2}, \ldots, \mathbf{h}_{k, N_{\mathrm{RF}}}\right]$, where $\mathbf{h}_{k, i}$ denotes the channel gain from the $i$ th subarray antennas to the $k$ th user. The analog precoding for the subarray $i$ at the $k$ th user can be selected as

$$
\mathbf{f}_{k}^{\star}=\underset{\mathbf{f}_{k} \in \mathcal{A}}{\arg \max }\left|\mathbf{h}_{k, i} \mathbf{f}_{k}\right|^{2} .
$$

We summarize the analog precoding selection scheme as Algorithm 1.

\section{B. Optimizing Digital Precoding and Power Splitting Ratio}

After obtaining the analog precoding $\mathbf{F}$, we define the equivalent channel as $\hat{\mathbf{h}}_{k}=\mathbf{h}_{k} \mathbf{F}$. By introducing auxiliary variables $t_{0}$ and $t_{k}$, the original EE maximization problem can be equivalently expressed as

$$
\begin{array}{ll} 
& \max _{\left\{\left\{\beta_{k}\right\},\left\{\mathbf{v}_{k}\right\}, t_{0},\left\{t_{k}\right\}\right\}} \frac{\sum_{k=0}^{K} \log _{2}\left(1+t_{k}\right)}{P_{\text {total }}} \\
\text { s.t. } & \frac{\beta_{k}\left|\hat{\mathbf{h}}_{k} \mathbf{v}_{0}\right|^{2}}{\beta_{k}\left(\sum_{i=1}^{K}\left|\hat{\mathbf{h}}_{k} \mathbf{v}_{i}\right|^{2}+\delta_{0}^{2}\right)+\delta_{1}^{2}} \geq t_{0}, k \in \mathcal{K}, \\
& \frac{\beta_{k}\left|\hat{\mathbf{h}}_{k} \mathbf{v}_{k}\right|^{2}}{\beta_{k}\left(\sum_{i \neq k}^{K}\left|\hat{\mathbf{h}}_{k} \mathbf{v}_{i}\right|^{2}+\delta_{0}^{2}\right)+\delta_{1}^{2}} \geq t_{k}, k \in \mathcal{K}, \\
& \varepsilon\left(1-\beta_{k}\right)\left(\sum_{i=0}^{K}\left|\hat{\mathbf{h}}_{k} \mathbf{v}_{i}\right|^{2}+\delta_{0}^{2}\right) \geq E_{k}^{\min }, k \in \mathcal{K}, \\
& \sum_{k=0}^{K}\left\|\mathbf{F} \mathbf{v}_{k}\right\|^{2} \leq P_{\max },
\end{array}
$$

It is evident that (15) is a nonconvex optimization problem due to the fractional objective function (15a) and nonconvex constraints (15b)-(15d). To solve the above problem, we first equivalently transform the fractional objection function into the subtractive form. We denote $q^{\star}$ as the obtained maximum EE of the system, namely

$q^{\star}=\frac{\sum_{k=0}^{K} \log _{2}\left(1+t_{k}^{\star}\right)}{\sum_{k=0}^{K} \xi\left\|\mathbf{F} \mathbf{v}_{k}^{\star}\right\|^{2}+P_{\mathrm{C}}}=\max _{\left\{\left\{\beta_{k}\right\},\left\{\mathbf{v}_{k}\right\}, t_{0},\left\{t_{k}\right\}\right\}} \frac{\sum_{k=0}^{K} \log _{2}\left(1+t_{k}\right)}{\sum_{k=0}^{K} \xi\left\|\mathbf{F} \mathbf{v}_{k}\right\|^{2}+P_{\mathrm{C}}}$,

where $\left\{\left\{\beta_{k}\right\},\left\{\mathbf{v}_{k}\right\}, t_{0},\left\{t_{k}\right\}\right\}$ should satisfy constraints (15b)-(15e). Then, we apply the following Theorem. 
Theorem 1: The maximum EE $q^{\star}$ is obtained if an only if

$$
\begin{gathered}
\max _{\left\{\left\{\beta_{k}\right\},\left\{\mathbf{v}_{k}\right\}, t_{0},\left\{t_{k}\right\}\right\}} \sum_{k=0}^{K} \log _{2}\left(1+t_{k}\right)-q^{\star}\left(\sum_{k=0}^{K} \xi\left\|\mathbf{F} \mathbf{v}_{k}\right\|^{2}+P_{\mathrm{C}}\right) \\
=\sum_{k=0}^{K} \log _{2}\left(1+t_{k}^{\star}\right)-q^{\star}\left(\sum_{k=0}^{K} \xi\left\|\mathbf{F} \mathbf{v}_{k}^{\star}\right\|^{2}+P_{\mathrm{C}}\right)=0
\end{gathered}
$$

where $\sum_{k=0}^{K} \log _{2}\left(1+t_{k}\right) \geq 0$ and $\sum_{k=0}^{K} \xi\left\|\mathbf{F} \mathbf{v}_{k}\right\|^{2}+P_{\mathrm{C}}>0$.

Proof The proof of Theorem 1 can follow the similar approach to the one in [18].

As a result, we need to solve the following optimization problem for a given $q$

$$
\max _{\left\{\left\{\beta_{k}\right\},\left\{\mathbf{v}_{k}\right\}, t_{0},\left\{t_{k}\right\}\right\}} \sum_{k=0}^{K} \log _{2}\left(1+t_{k}\right)-q \sum_{k=0}^{K} \xi\left\|\mathbf{F} \mathbf{v}_{k}\right\|^{2}
$$

s.t. $(15 b)-(15 e)$,

where $q$ can be regarded as a parameter and we can denote the optimal value of (18a) as $T(q)$. To this end, we have the following according to Theorem 1

$$
q=q^{\star} \Leftrightarrow T(q)=0,
$$

which means that searching the root for the nonlinear equation $T(q)=0$ is equivalent to solve (15). It can be found that $T(q)$ is a strictly decreasing and convex function with respect to $q$, where $T(q)>0$ with $q \rightarrow-\infty$ and $T(q)<0$ with $q \rightarrow \infty$. Therefore, we can use the classical Bi-section method to find $T(q)=0$, which is summarized as Algorithm 2 .

Apparently, (18) is a nonconvex optimization problem due to the nonconvex constraints $(15 b)-(15 d)$. Next, we define two new variables $\mu_{k}=1 / \beta_{k}$ and $\omega_{k}=1 /\left(1-\beta_{k}\right)$, and then we reformulate the following optimization problem

$$
\begin{gathered}
\max _{\left\{\left\{\mu_{k}\right\},\left\{\omega_{k}\right\},\left\{\mathbf{v}_{k}\right\}, t_{0},\left\{t_{k}\right\}\right\}} \sum_{k=0}^{K} \log _{2}\left(1+t_{k}\right)-q \sum_{k=0}^{K} \\
\left|\hat{\mathbf{h}}_{k} \mathbf{v}_{0}\right|^{2} \geq t_{0}\left(\sum_{i=1}^{K}\left|\hat{\mathbf{h}}_{k} \mathbf{v}_{i}\right|^{2}+\delta_{0}^{2}+\mu_{k} \delta_{1}^{2}\right), k \in \mathcal{K}, \\
\left|\hat{\mathbf{h}}_{k} \mathbf{v}_{k}\right|^{2} \geq t_{k}\left(\sum_{i \neq k}^{K}\left|\hat{\mathbf{h}}_{k} \mathbf{v}_{i}\right|^{2}+\delta_{0}^{2}+\mu_{k} \delta_{1}^{2}\right), k \in \mathcal{K}, \\
\sum_{i=0}^{K}\left|\hat{\mathbf{h}}_{k} \mathbf{v}_{i}\right|^{2}+\delta_{0}^{2} \geq \frac{E_{k}^{\min }}{\varepsilon} \omega_{k}, k \in \mathcal{K}, \\
\left\|\begin{array}{l}
\mu_{k}-\omega_{k} \\
2
\end{array}\right\|_{2} \leq \mu_{k}+\omega_{k}-2, k \in \mathcal{K},
\end{gathered}
$$

(15e).

Meanwhile, we have the following theorem.

Theorem 2: The optimal solution of (20) is also the optimal solution of (18).

Proof From (20e), we have $\mu_{k}+\omega_{k} \leq \mu_{k} \omega_{k}$ and $\frac{1}{\mu_{k}}+\frac{1}{\omega_{k}} \leq 1$. Assume $\left\{\left\{\mu_{k}^{\star}\right\},\left\{\omega_{k}^{\star}\right\},\left\{\mathbf{v}_{k}^{\star}\right\}, t_{0}^{\star},\left\{t_{k}^{\star}\right\}\right\}$ represents a global optimization solution of (20). If (20e) is satisfied with equality, namely $1 / \mu_{k}^{\star}+1 / \omega_{k}^{\star}=1$, it is clear that problems (18) and (20) are equivalent and we only need to replace $1 / \mu_{k}^{\star}$ with $\beta_{k}^{\star}$ for $k \in \mathcal{K}$. Otherwise, if $1 / \mu_{k}^{\star}+1 / \omega_{k}^{\star}<1$, we scale $\left\{\left\{\mu_{k}^{\star}\right\},\left\{\omega_{k}^{\star}\right\}\right\}$

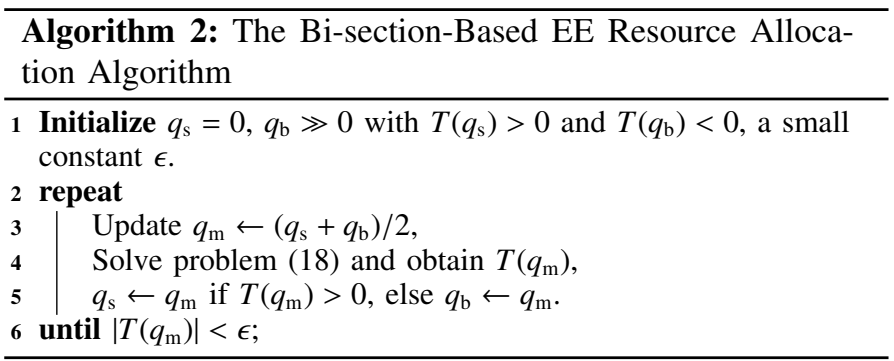

by $\left(1 / \mu_{k}^{\star}+1 / \omega_{k}^{\star}\right)$ and have

$$
\frac{1}{\mu_{k}^{\star}\left(1 / \mu_{k}^{\star}+1 / \omega_{k}^{\star}\right)}+\frac{1}{\omega_{k}^{\star}\left(1 / \mu_{k}^{\star}+1 / \omega_{k}^{\star}\right)}=1 .
$$

It means that (20e) can satisfy the equality and the harvested power does not violate the constraint (20d) due to $1 / \mu_{k}^{\star}+1 / \omega_{k}^{\star}<1$. As a result, the values of $\sum_{i=1}^{K}\left|\hat{\mathbf{h}}_{k} \mathbf{v}_{i}\right|^{2}+\delta_{0}^{2}+\mu_{k} \delta_{1}^{2}$ and $\sum_{i \neq k}^{K}\left|\hat{\mathbf{h}}_{k} \mathbf{v}_{k}\right|^{2}+\delta_{0}^{2}+\mu_{k} \delta_{1}^{2}$ in (20b) and (20c) will become smaller. Meanwhile, we can obtain a large $\left\{t_{0}, t_{k}\right\}$ than the optimal $\left\{t_{0}^{\star}, t_{k}^{\star}\right\}$, which means a greater rate can be obtained for the same $\mathbf{v}_{k}$. It is contradictory with our original assumption, and we finish the proof.

Next, we need to solve (20), which is still a nonconvex optimization problem due to the nonconvex constraints (20b)(20d). Let $\left\{\hat{\mathbf{v}}_{k}\right\}(k \in \mathcal{K})$ is a feasible solution and then, we define $\mathbf{v}_{k} \triangleq \hat{\mathbf{v}}_{k}+\Delta \mathbf{v}_{k}$ and have

$$
\begin{aligned}
\left|\hat{\mathbf{h}}_{k} \mathbf{v}_{k}\right|^{2} & =\left(\hat{\mathbf{v}}_{k}+\Delta \mathbf{v}_{k}\right)^{H} \hat{\mathbf{H}}_{k}\left(\hat{\mathbf{v}}_{k}+\Delta \mathbf{v}_{k}\right) \\
& \geq 2 \operatorname{Re}\left\{\left(\hat{\mathbf{v}}_{k}\right)^{H} \hat{\mathbf{H}}_{k} \Delta \mathbf{v}_{k}\right\}+\left(\hat{\mathbf{v}}_{k}\right)^{H} \hat{\mathbf{H}}_{k} \hat{\mathbf{v}}_{k},
\end{aligned}
$$

where $\hat{\mathbf{H}}_{k}=\hat{\mathbf{h}}_{k}^{H} \hat{\mathbf{h}}_{k}$. In this case, $\left|\hat{\mathbf{h}}_{k} \mathbf{v}_{k}\right|^{2}$ can be replaced by its convex approximations and the formulated problem can be solved iteratively. Accordingly, (20b)-(20d) can transform as

$$
\begin{array}{r}
2 \operatorname{Re}\left\{\left(\hat{\mathbf{v}}_{0}\right)^{H} \hat{\mathbf{H}}_{k} \Delta \mathbf{v}_{0}\right\}+\left(\hat{\mathbf{v}}_{0}\right)^{H} \hat{\mathbf{H}}_{k} \hat{\mathbf{v}}_{0} \geq t_{0}\left(\sum_{i=1}^{K}\left|\hat{\mathbf{h}}_{k} \mathbf{v}_{i}\right|^{2}+\delta_{0}^{2}+\mu_{k} \delta_{1}^{2}\right) \\
2 \operatorname{Re}\left\{\left(\hat{\mathbf{v}}_{k}\right)^{H} \hat{\mathbf{H}}_{k} \Delta \mathbf{v}_{k}\right\}+\left(\hat{\mathbf{v}}_{k}\right)^{H} \hat{\mathbf{H}}_{k} \hat{\mathbf{v}}_{k} \geq t_{k}\left(\sum_{i \neq k}^{K}\left|\hat{\mathbf{h}}_{k} \mathbf{v}_{i}\right|^{2}+\delta_{0}^{2}+\mu_{k} \delta_{1}^{2}\right) \\
\sum_{i=0}^{K} 2 \operatorname{Re}\left\{\left(\hat{\mathbf{v}}_{i}\right)^{H} \hat{\mathbf{H}}_{k} \Delta \mathbf{v}_{i}\right\}+\left(\hat{\mathbf{v}}_{i}\right)^{H} \hat{\mathbf{H}}_{k} \hat{\mathbf{v}}_{i}+\delta_{0}^{2} \geq E_{k}^{\min } \omega_{k} / \varepsilon .
\end{array}
$$

Then, we set the new variables $\tau_{k} \geq \sum_{i=1}^{K}\left|\hat{\mathbf{h}}_{k} \mathbf{v}_{i}\right|^{2}+\delta_{0}^{2}+\mu_{k} \delta_{1}^{2}$ and $\lambda_{k} \geq \sum_{i \neq k}^{K}\left|\hat{\mathbf{h}}_{k} \mathbf{v}_{i}\right|^{2}+\delta_{0}^{2}+\mu_{k} \delta_{1}^{2}$, and reformulate the following optimization as

$\max _{\left\{\left\{\mu_{k}\right\},\left\{\omega_{k}\right\},\left\{\tau_{k}\right\},\left\{\lambda_{k}\right\},\left\{\mathbf{v}_{k}\right\}, t_{0},\left\{t_{k}\right\}\right\}} \sum_{k=0}^{K} \log _{2}\left(1+t_{k}\right)-q \sum_{k=0}^{K} \xi\left\|\mathbf{F v}_{k}\right\|^{2}$

s.t. $2 \operatorname{Re}\left\{\left(\hat{\mathbf{v}}_{0}\right)^{H} \hat{\mathbf{H}}_{k} \Delta \mathbf{v}_{0}\right\}+\left(\hat{\mathbf{v}}_{0}\right)^{H} \hat{\mathbf{H}}_{k} \hat{\mathbf{v}}_{0} \geq t_{0} \tau_{k}, k \in \mathcal{K}$,

$\sum_{i=1}^{K}\left|\hat{\mathbf{h}}_{k} \mathbf{v}_{i}\right|^{2}+\delta_{0}^{2}+\mu_{k} \delta_{1}^{2} \leq \tau_{k}, k \in \mathcal{K}$,

$2 \operatorname{Re}\left\{\left(\hat{\mathbf{v}}_{k}\right)^{H} \hat{\mathbf{H}}_{k} \Delta \mathbf{v}_{k}\right\}+\left(\hat{\mathbf{v}}_{k}\right)^{H} \hat{\mathbf{H}}_{k} \hat{\mathbf{v}}_{k} \geq t_{k} \lambda_{k}, k \in \mathcal{K}$,

$\sum_{i \neq k}^{K}\left|\hat{\mathbf{h}}_{k} \mathbf{v}_{i}\right|^{2}+\delta_{0}^{2}+\mu_{k} \delta_{1}^{2} \leq \lambda_{k}, k \in \mathcal{K}$,

(15e), (20e), (25). 


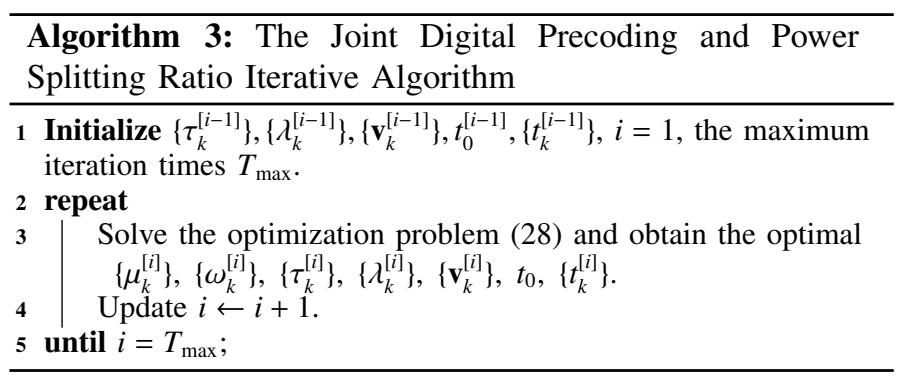

So far, the only nonconvex constraints are (26b) and (26d) in (26). Similar to [19], the upper of $t_{0} \tau_{k}$ and $t_{k} \lambda_{k}$ can be written as

$$
\frac{t_{0}^{[i-1]}}{2 \tau_{k}^{[i-1]}} \tau_{k}^{2}+\frac{\tau_{k}^{[i-1]}}{2 t_{0}^{[i-1]}} t_{0}^{2} \geq t_{0} \tau_{k}, \quad \frac{t_{k}^{[i-1]}}{2 \lambda_{k}^{[i-1]}} \lambda_{k}^{2}+\frac{\lambda_{k}^{[i-1]}}{2 t_{k}^{[i-1]}} t_{k}^{2} \geq t_{k} \lambda_{k},
$$

where $\left\{t_{0}^{[i-1]},\left\{\tau_{k}^{[i-1]}\right\},\left\{t_{k}^{[i-1]}\right\},\left\{\lambda_{k}^{[i-1]}\right\}\right\} \quad$ are the value of $\left\{t_{0},\left\{\tau_{k}\right\},\left\{t_{k}\right\},\left\{\lambda_{k}\right\}\right\}$ at the $(i-1)$ th iteration. Finally, we formulate the following optimization problem

$$
\begin{array}{r}
\max _{\left\{\left\{\mu_{k}\right\},\left\{\omega_{k}\right\},\left\{\tau_{k}\right\},\left\{\lambda_{k}\right\},\left\{\mathbf{v}_{k}\right\}, t_{0},\left\{t_{k}\right\}\right\}} \sum_{k=0}^{K} \log _{2}\left(1+t_{k}\right)-q \sum_{k=0}^{K} \xi\left\|\mathbf{F} \mathbf{v}_{k}\right\|^{2} \\
\text { s.t. } 2 \operatorname{Re}\left\{\left(\hat{\mathbf{v}}_{0}\right)^{H} \hat{\mathbf{H}}_{k} \Delta \mathbf{v}_{0}\right\}+\left(\hat{\mathbf{v}}_{0}\right)^{H} \hat{\mathbf{H}}_{k} \hat{\mathbf{v}}_{0} \geq \frac{t_{0}^{[i-1]}}{2 \tau_{k}^{[i-1]}} \tau_{k}^{2}+\frac{\tau_{k}^{[i-1]}}{2 t_{0}^{[i-1]}} t_{0}^{2}, \\
2 \operatorname{Re}\left\{\left(\hat{\mathbf{v}}_{k}\right)^{H} \hat{\mathbf{H}}_{k} \Delta \mathbf{v}_{k}\right\}+\left(\hat{\mathbf{v}}_{k}\right)^{H} \hat{\mathbf{H}}_{k} \hat{\mathbf{v}}_{k} \geq \frac{t_{k}^{[i-1]}}{2 \lambda_{k}^{[i-1]}} \lambda_{k}^{2}+\frac{\lambda_{k}^{[i-1]}}{2 t_{k}^{[i-1]}} t_{k}^{2},
\end{array}
$$

(15e), (20e), (25), (26c), (26e).

It is clear that (28) is convex optimization problem due to the concave objection function (28a) and convex constraints (28b)-(28d), which can be solve by existing convex solvers (e.g., CVX). Summarily, solving the original problem (18), we need to iteratively solve the optimal values of $\left\{\left\{\mu_{k}\right\},\left\{\omega_{k}\right\},\left\{\tau_{k}\right\},\left\{\lambda_{k}\right\},\left\{\mathbf{v}_{k}\right\}, t_{0},\left\{t_{k}\right\}\right\}$ via (28). In addition, since obtained $\left\{\left\{\mu_{k}^{[i]}\right\},\left\{\omega_{k}^{[i]}\right\},\left\{\tau_{k}^{[i]}\right\},\left\{\lambda_{k}^{[i]}\right\},\left\{\mathbf{v}_{k}^{[i]}\right\}, t_{0}^{[i]},\left\{t_{k}^{[i]}\right\}\right\}$ are the optimal solutions at the $i$ th iteration, iteratively updating these variables will increase or maintain the value of the objective function (28a). To this end, the proposed iterative algorithm will converge to at least a local optimal solution, which is summarized in Algorithm 3.

\section{Simulation Results}

In this section, simulation results are provided to illustrate the effectiveness of the proposed algorithms. We assume that the BS has a coverage of 30 meters, and the path loss is modeled as $69.4+24 \log _{10}(d) \mathrm{dB}$, where $d$ denotes the distance in meter. We assume that there are 8 paths for the mmWave channel, and the azimuth angle of departure at BS is uniformly distributed over $[0,2 \pi]$. The BS is equipped with $N_{\mathrm{TX}}=256$ antennas and $N_{\mathrm{RF}}=4 \mathrm{RF}$ chains. The noise power, $\delta_{0}^{2}$ and $\delta_{1}^{2}$, are set $-80 \mathrm{dBm}$ and $-60 \mathrm{dBm}$, respectively. The energy conversion efficiency $\varepsilon$ and the inefficiency of the power amplifier $\xi$ are set as 0.5 and $1 / 0.38$, respectively. In addition, we set $P_{\mathrm{BB}}=200$ $\mathrm{mW}, P_{\mathrm{RF}}=300 \mathrm{~mW}, P_{\mathrm{PS}}=40 \mathrm{~mW}$. Meanwhile, the minimum harvested energy is $E_{k}^{\min }=100 \mu \mathrm{W}$ for all users, the number of users is set as $K=2$.

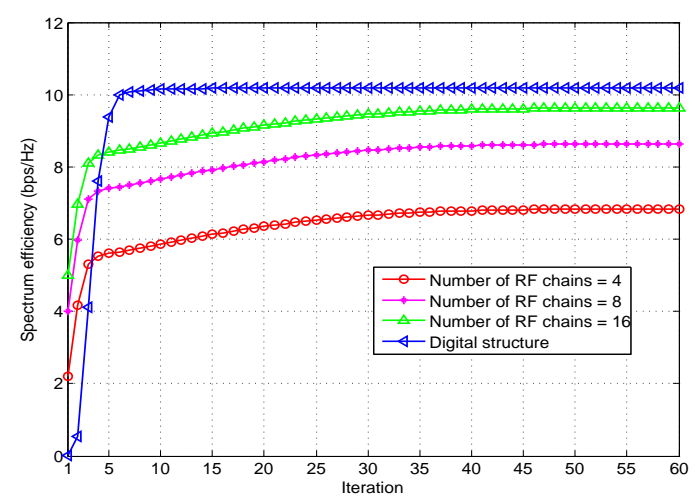

Fig. 3: Spectrum efficiency versus number of iterations.

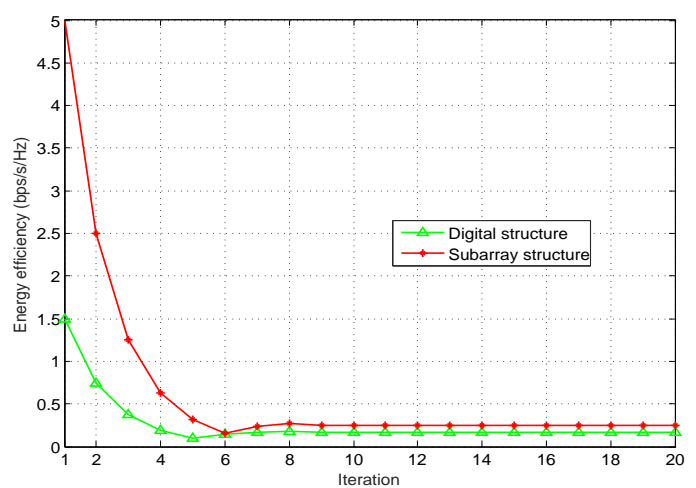

Fig. 4: Energy efficiency versus number of iterations.

Fig. 3 shows the performance of convergence for the proposed Algorithms 3 under different number of RF chains, where digital structure means that each antenna is connected to one dedicated RF chain. We set $q=0$ and the maximum transmit power $P_{\max }=30 \mathrm{dBm}$. It is clear that the proposed algorithm will converge after multiple iterations. In addition, one can observe that the SE under digital structure is the highest in comparison with that under subarray structure.

Fig. 4 shows the convergence property for the Algorithm 2, where we set the maximum transmit power $P_{\max }=40 \mathrm{dBm}$. Meanwhile, we compare the EE under different number of RF chains. It can be observed that the proposed algorithm will converge about 8 iterations. In addition, one can see that the EE with subarray structure is higher than that with digital structure. This is because although the SE with digital structure is higher as shown in Fig. 3, the circuit's power consumption is huge.

Fig. 5 shows the EE versus maximum transmit power at BS. We can observe that the EE first increases and then remains stable with $P_{\text {max }}$. Understandably, larger transmit power can improve the SE of the system, but the rate of the SE will be lower. Therefore, the EE will reach the point of diminishing returns when the transmit power continues to increase. In addition, it is clear that the EE with sparse RF chains is higher just as explained earlier, and the digital structure still has a huge circuit's power consumption.

We examine the EE of the system under different optimiza- 


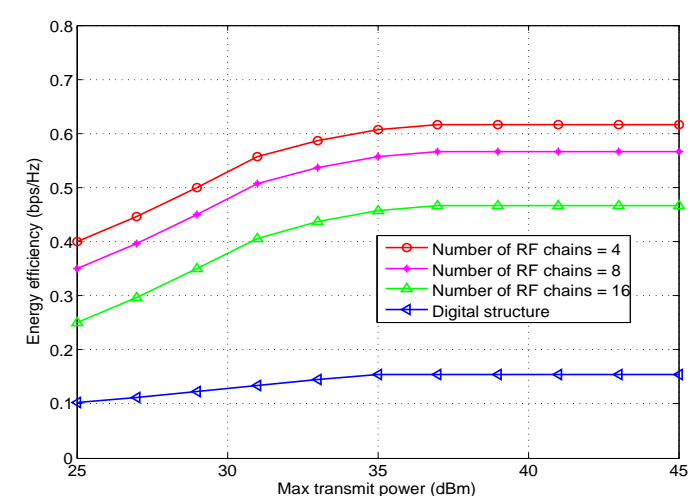

Fig. 5: Energy efficiency versus max transmit power.

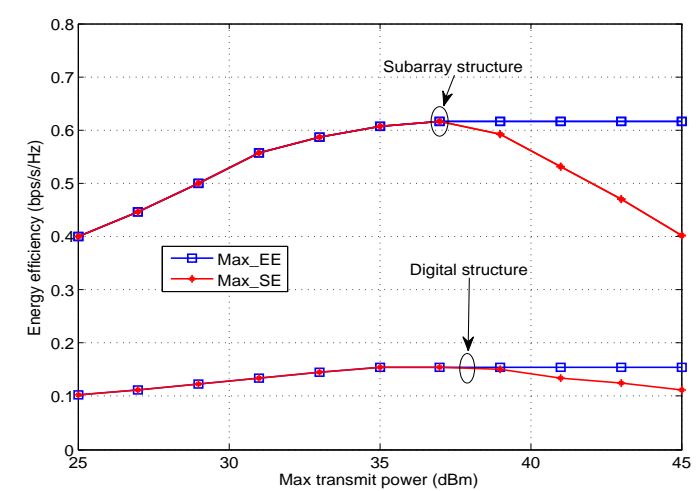

Fig. 6: Energy efficiency versus max transmit power.

tion schemes in Fig. 6. "Max_EE" stands that the EE of the system when the EE is maximized, while "Max SE" represents the EE of the system when the SE is maximized. As the maximum transmit power increases, the EE reaches maximum and remains constant under "Max EE" scheme, while the EE decreases under "Max SE" scheme. In fact, the objection of "Max_SE" scheme is to maximize the SE without considering the power consumption. As a result, the EE may decrease for larger transmit power.

\section{Conclusions}

In this paper, we investigated the EE maximization problem in a joint multicast-unicast mmWave communication system with SWIPT. We first designed the analog precoding for the sparse RF chain structure. Next, we proposed a two-loop algorithm to solve the formulated EE optimization problem. The Bi-section algorithm is adopted in outer loop. Subsequently, we developed an iterative algorithm for the inner loop. Simulation

\section{AcKnowledgment}

This work is supported in part by the European Commission under the 5GPPP project 5GXcast (H2020-ICT-20162 call, grant number 761498), and in part by the U.K. Engineering and Physical Sciences Research Council under results showed the effectiveness of the proposed algorithm, and there still a tradeoff between EE and SE.

Grant EP/P008402/1, and in part by the National Natural Science Foundation of China (Grant:61701214), and in part by the Excellent Youth Foundation of Jiangxi Province (Grant 2018ACB21012), and in part by the China Postdoctoral Science Foundation (First rank) (Grant: 2017M610400). The authors also would like to acknowledge the support of the University of Surrey 5GIC (www.surrey.ac.uk/5gic) members for this work.

\section{REFERENCES}

[1] X. Gao, et al., "Low RF-complexity technologies to enable millimeterwave MIMO with large antenna array for $5 \mathrm{G}$ wireless communications," IEEE Commun. Mag., vol. 56, no. 4, pp. 211-217, Apr. 2018.

[2] W. Hao, et al., "Price-based resource allocation in massive MIMO HCRANs with limited fronthaul capacity," IEEE Trans. Wireless Commun., vol. 17, no. 11, pp. 7691-7703, Nov. 2018.

[3] R. W. Heath et al., "An overview of signal processing techniques for millimeter wave MIMO systems," IEEE J. Sel. Top. Signal Process., vol. 10, no. 3, pp. 436-53, Apr. 2016.

[4] W. Hao, et al., "Energy-efficient power allocation in millimeter wave massive MIMO with non-orthogonal multiple access," IEEE Wireless Commun. Lett., vol. 6, no. 6, pp. 782-785, Dec. 2017.

[5] F. Zhou, et al., "Robust AN-aided beamforming and power splitting design for secure MISO cognitive radio with SWIPT,' IEEE Trans. Wireless Commun., vol. 16, no. 4, pp. 2450-2464, April 2017.

[6] Q. Shi, et al., "Joint transmit beamforming and receive power splitting for MISO SWIPT systems," IEEE Trans. Wireless Commun., vol. 13, no. 6, pp. 3269-3280, Jun. 2014.

[7] Z. Ding, et al.,"On the spectral efficiency and security enhancements of NOMA assisted multicast-unicast streaming," IEEE Trans. Commun., vol. 65, no. 7, pp. 3151-3163, Jul. 2017.

[8] E. G. Larsson, et al., "Joint beamforming and broadcasting in massive MIMO," IEEE Trans. Wireless Commun., vol. 15, no. 4, pp. 3058-3070, Apr. 2016.

[9] B. Xu, et al., "Optimized power allocation for interference channel with SWIPT," IEEE Wireless Commun. Lett., vol. 5, no. 2, pp. 220-223, Apr. 2016.

[10] O. T. Demir, et al., "Maxmin fair resource allocation for SWIPT in multigroup multicast OFDM systems," IEEE Commun. Lett., vol. 21, no. 11, pp. 2508-2511, Nov. 2017.

[11] O. T. Demir, et al., "SWIPT for max-min fair multi-group multicast beamforming through power splitting," IEEE WCNC, 2017, pp. 1-6.

[12] O. T. Demir, et al., "Antenna selection and hybrid beamforming for simultaneous wireless information and power transfer in multi-group multicasting systems," IEEE Trans. Wireless Commun., vol. 15, no. 10, pp. 6948-6962, Oct. 2016.

[13] B. Wang, et al., "Spectrum and energy-efficient beamspace MIMONOMA for millimeter-wave communications using lens antenna array," IEEE J. Sel. Areas Commun., vol. 35, no. 10, pp. 2370-2382, Oct. 2017.

[14] W. Hao, et al., "Energy-efficient resource allocation for mmWave massive MIMO HetNets with wireless backhaul," IEEE Access, vol. 6, pp. 24572471, 2018.

[15] J. Zhao, et al., "Nonorthogonal unicast and broadcast transmission via joint beamforming and ldm in cellular networks," IEEE Global Commun. Conf. (GLOBECOM), Dec. 2016, pp. 1-6.

[16] W. Hao, et al., "Small cell cluster-based resource allocation for wireless backhaul in two-tier heterogeneous networks with massive MIMO," IEEE Trans. Veh. Technol., vol. 67, no. 1, pp. 509-523, Jan. 2018.

[17] W. Hao, et al., "Green communication for NOMA-based CRAN," IEEE Internet of Things Journal, vol. 6, no. 1, pp. 666-678, Feb. 2019.

[18] W. Dinkelbach, "On nonlinear fractional programming," Management Science, vol. 13, pp. 492-498, Mar. 1967. Available: http://www.jstor.org/stable/2627691

[19] P. Song, et al., "D3M: Distributed multi-cell multigroup multicasting," in 2016 IEEE Int. Conf. Acoustics, Speech and Signal Processing (ICASSP), Shanghai, 2016, pp. 3741-3745. 